\section{Befunde auf einen Blick}

Wer von uns kennt nicht die befriedigende rasche Einordnung von komplizierten Befunden? Ein geschulter diagnostischer Blick ist auch in einer hoch differenzierten und technisierten Medizin von hohem Wert. Tischendorf und seine Mitautoren zeigen uns in ihrem Atlas und Textbuch häufig auftretende wie auch seltene Krankheitsbilder aus allen Feldern der Medizin. Die überarbeitete 7. Auflage wurde durch über 300 neue Abbildungen und zahlreiche differenzialdiagnostische Zusatzinformationen ergänzt. Auch viele einprägsame Übersichtstabellen haben die Autoren eingefügt. Schon etwas veraltet ist dagegen die Klassifikation der Hämangiome.

Insgesamt bietet dieses Buch in seiner aktuellen Auflage aber wieder einen exzellenten Fundus für Ärzte jeder Fachrichtung.

Dr. Gesine Maevers

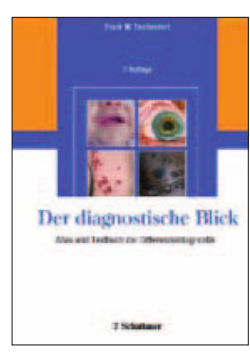

F. W. Tischendorf (Hrsg.) Der diagnostische Blick

Schattauer, Stuttgart 2008 99,00€ ISBN 978-3-79452474-7

\section{Frauen an die Front!}

Die Zukunft der Medizin ist weiblich: Von den Medizinstudierenden sind in Deutschland zurzeit über 60\% Frauen, bei den berufstätigen Medizinern machen in den Altersgruppen bis 39 Jahre die Ärztinnen bereits einen Anteil von fast $50 \%$ aus. Gleichzeitig ist die Zahl der Ärztinnen in Führungspositionen nach wie vor gering, aber das wird sich nach Einschätzung vieler Meinungsbildner in der nächsten Zeit ändern.

Ziel des hier vorgestellten Buches ist es, Frauen, die eine Führungsposition als Oberärztin oder Chefärztin anstreben, Hilfsmittel mit auf den Weg zu geben, wie sie diese erreichen können und wie sie gängige Fehler vermeiden können. Im „Führungshandbuch für Ärztinnen " findet sich eine Analyse der derzeitigen Lage, der Chancen und auch der Schwierigkeiten, die man als Frau in der Führungsriege der Ärzteschaft zu erwarten hat. Das Handbuch bietet konkrete Handlungsanweisungen, wie Führung im beruflichen Alltag umgesetzt werden kann, außerdem Erfahrungsberichte sowie Tipps und Tricks von Ärztinnen in Führungspositionen aus Deutschland, Österreich und der Schweiz. Die Handlungsanweisungen werden ergänzt durch Übungen und praktische Beispiele, die bei der Umsetzung gesunder Führung helfen.

red

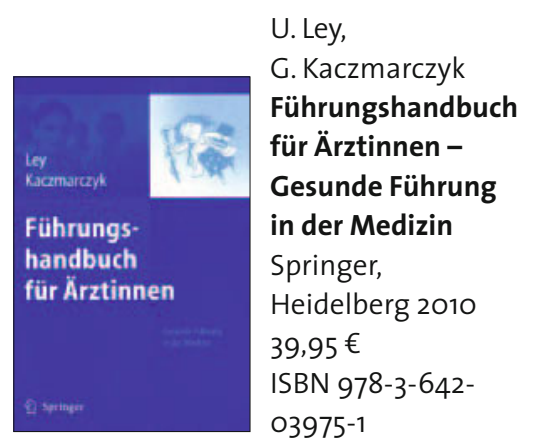

\section{In die Trickkiste gegriffen}

„Wir waren schon überall und keiner kann uns helfen. "Unterschwellig klingt mit: ,Sie sicher auch nicht." Wer kennt nicht diese Gesprächseröffnung und den Druck, den sie beim Behandler erzeugt? Anregungen, wie er darauf reagieren kann, findet der Kinderarzt in dem hier vorgestellten Buch des Ehepaars Caby. Er erfährt, wie er mit ganz überraschenden Interventionen sein Gegenüber verblüffen, irritieren und zum Mitarbeiten animieren kann. Dabei profitiert der Leser von der profunden praktischen Erfahrung der Autoren, er Facharzt für Kinder- und Jugendpsychiatrie und -psychotherapie, sie Fachärztin für Kinderheilkunde und Jugendmedizin.

Nach einer kurzen theoretischen Einleitung werden zahlreiche Interventionsmöglichkeiten vorgestellt und im Hinblick auf ihren Sinn und ihre Anwendung diskutiert. Das Buch endet mit dem Kapitel „Indikationen oder: Was hilft wann am besten?“. Hier werden häufige Symptome und Krankheitsbilder aufgelistet, bei denen sich ein bestimmtes Vorgehen als besonders wirksam erwiesen hat.

Einige der „Tipps und Tricks“ in dem Buch hat der Leser sicher schon intuitiv mit Erfolg eingesetzt, sie aber vielleicht wieder vergessen. In dem Fall wird er das Aha-Erlebnis des Wiedererkennens haben. Viele der Techniken sind jedoch neu und machen Lust, sie in die Praxis umzusetzen. So hat mein Patient Carsten begeistert auf das Spiel „Veränderungsdetektiv“ reagiert: Eltern und Kind müssen ein unerwünschtes Verhalten verändern oder ein erwünschtes Verhalten zeigen - und der jeweils andere muss erraten, um welche Verhaltensweise es geht. Ich bin gespannt auf das nächste Treffen, bei dem Carsten mir erzählen wird, was er bei seiner Mutter beobachtet hat, und seine Mutter über ihre Eindrücke berichten wird - und was sich sonst noch alles bei den beiden verändert hat. Auch die Frage „Warum darf der das?“ werde ich sicher häufiger einsetzen, wenn sich wieder einmal eine Mutter über das Verhalten ihres Kindes beklagt.

Dem Anspruch, ein kurzweiliges Buch anzubieten, das gern gelesen und in dem immer wieder nachgeblättert wird, sind die Autoren zweifellos gerecht geworden: Es soll kein dickes Buch sein, sondern eins, das genutzt wird. Meine Arbeit ist durch die Lektüre wieder spannender geworden, weil ich jetzt Neues ausprobieren kann und neugierig bin, was sich dadurch verändern wird. Das Buch ist für niedergelassene Kinder- und Jugendärzte sehr zu empfehlen, da viele in dem Buch angesprochenen Bereiche wie Enuresis, Enkopresis und Schlafstörungen zu ihrem Praxisalltag gehören und ihnen Anregungen vermittelt werden, wie sie ihre Patienten und deren Eltern unterstützen und aus der therapeutischen Sackgasse führen können.

\section{Dr. Kirsten Stollhoff}

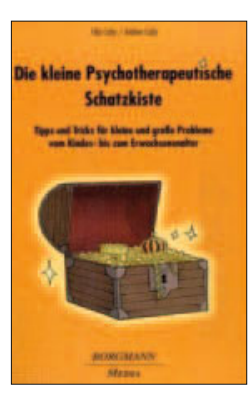

F. Caby, A. Caby Die kleine Psychotherapeutische Schatzkiste Verlag Modernes Lernen, Dortmund 2009 $19,95 €$ ISBN 978-3-93818747-0 